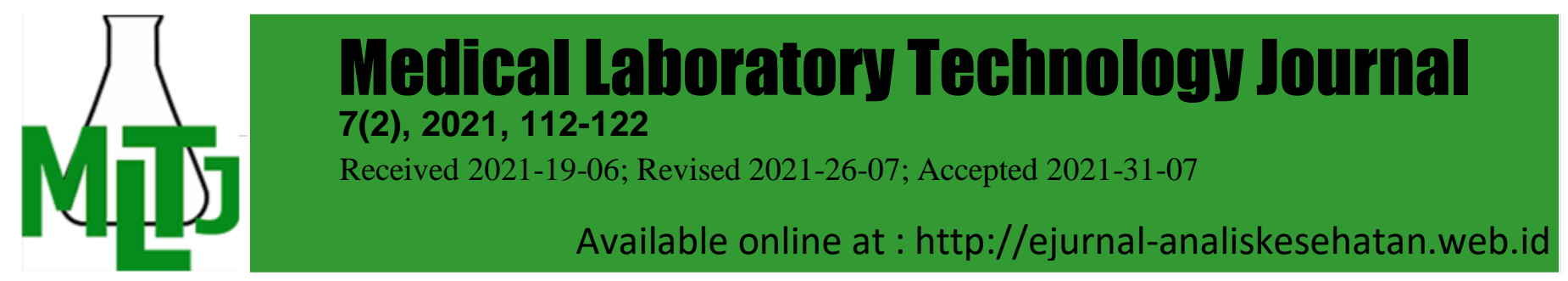

\title{
The Place of Histochemical Stains for Differential Diagnosis in Duodenal Biopsies
}

\author{
*Ebru Altındal Susem¹, Mehtap Eroğlu², Elif Acar ${ }^{1}$, İrfan Bayram ${ }^{3}$ \\ 1Department of Pathology, Niğde Ömer Halis Demir training and research Hospital, \\ Ömer Halis Demir university medical faculty, Niğde, Turkey. ${ }^{2}$ Department of \\ Pathology, City hospital, Adana, Turkey. ${ }^{3}$ Prof Dr İrfan Bayram Pathology \\ Laboratory, Van, Turkey. *Email: ebruasusem@gmail.com \\ DOI: $10.31964 / \mathrm{mltj} . \mathrm{v} 0 \mathrm{i0} 0.385$
}

\begin{abstract}
The biopsy is the primary examination method in diagnosing duodenal pathologies and Gluten enteropathy that can cause symptoms such as gas, diarrhea, weight loss, anemia, osteoporosis. Allows monitoring of response to therapy. Histochemical studies are inevitable in specific findings such as reactive changes, giardia, subepithelial collagen thickening, fibrosis, and gastric metaplasia. We aimed to understand the contribution of histochemical applications to differential diagnosis and to show their superiority compared to hematoxylin-eosin sections in routine use by simultaneously making histomorphological evaluations on hematoxylin and eosin sections. This research is a retrospective study conducted in 2011, using the archives of Yüzüncü Yıl University, Faculty of Medicine, Department of Pathology, between 2001-2010. Ten normal, 50 Gluten enteropathy and 50 duodenitis samples; Along with histopathological examinations, we applied Masson Trichrome, Periodic acid-Schiff, Periodic acid-Schiff with diastasis, Periodic acid Schiff-Alcian Blue, High Iron DiamineAlcian Blue. Chi-square, likelihood ratio tests, and SPSS (ver: 13) statistical package program used. We detected microscopic findings parallel to diagnostic criteria. While the number of goblet cells and intraepithelial neutrophils did not differ in the groups $(P$ $=0.176$ and $P=0.096$ ), there was a significant variation in the intraepithelial lymphocyte count $(P=0.010)$. The frequency of flattening and blunting of the villi, crypt hyperplasia, and Brunner gland hyperplasia were significant in gluten enteropathies $(P=0.000)$. Conclusion We could not find any difference in specific pathologies associated with histochemical studies in our group, so hematoxylin-eosin sections are more valuable in the differential diagnosis.
\end{abstract}

Keywords: Duodenum; gluten enteropathy; histochemical staining

\section{INTRODUCTION}

The primary function of the duodenum, which is the first and shortest part of the small intestine between the stomach and the jejunum, is to receive food partially digested by the stomach and complete the digestion process. The duodenum provides the necessary digestion for food with fluids from the pancreas, gall bladder, and liver. After digestion completes, columnar cells absorb substances such as proteins, carbohydrates, and lipids. Duodenum regulates the bile secretion of the gall bladder, the exocrine secretion of the pancreas, and the stomach's movements by the secretin and cholecystokinin secreted by it (Johnson, 2013). Due to some deterioration in duodenal functions, substantial health problems such as excessive gas, diarrhea, weight loss, iron deficiency anemia, and osteoporosis occur. Although the clinical symptoms show a similar course in different pathologies, treatment methods differ. 
Duodenal mucosa must be evaluated histopathologically for correct treatment. Duodenitis is mucosal inflammation due to Helicobacter Pylori, peptic ulcers, Zollinger Ellison syndrome, gastritis, nonsteroidal anti-inflammatory drugs, Crohn's disease, and gastrointestinal bleeding (Noble et al., 2001). Aggressive factors such as $H$. pylori and ascites and chronic inflammation in the duodenal mucosa may cause gastric metaplasia. If $H$. pylori eradication does, alkaline secretion increases, mucosa regenerates, and gastric metaplasia regresses. It characterizes by duodenitis in hematoxylin-eosin sections, increased plasma cells, pronounced mucosal edema accompanied by increased polymorph in villi, especially crypt epithelium and lamina propria, and gastric metaplasia (figure 1). In severe duodenitis, villus atrophy and prominent polymorph infiltration to lamina propria, crypt, and villi epithelium occur. Polymorphs and gastric metaplasia may not see in all cases (Hasan et al., 1981).

Celiac Disease (gluten enteropathy) is an inherited disease that develops as a result of intolerance to gluten found in cereals such as wheat, barley, oats, and rye, progresses with histopathological findings characterized by inflammation and villus atrophy in the small intestine, and improves with a gluten-free diet (Farrell et al., 2002). The diagnosis makes while investigating the causes of anemia, osteoporosis, autoimmune disease, neurological syndromes of unknown cause, delayed puberty, nonspecific arthritis, depression, ataxia, gingival hyperplasia, infertility, and chronic hypertransemia in adulthood (Sanders et al., 2002). Genetic, environmental, and immunological factors play a role in the pathogenesis of the celiac disease. It knowns that in gluten enteropathy, there is mucosal damage due to overstimulation of cellular and humoral immunity, and gamma interferon ( $g$-INF) produced by gluten-specific $T$ cells is activated (Nilsen et al., 1998 and Przemioslo et al., 1995). Intraepithelial lymphocyte infiltration (IEL) is a critical feature in gluten enteropathy histopathology.

Giardia lamblia (Giardia intestinalis) is one of the most common pathogens among intestinal parasites worldwide, causing mostly asymptomatic infection. The protozoon constitutes the most common cause of chronic diarrhea in our country (Parasites and Health: Giardiasis, 2006). They are in the form of trophozoites and cysts. Infection is transmitted to humans when cyst forms are taken orally with water and food contaminated with eggs in the stool (Eichinger, 2001 and Gillin et al., 1996).

Giardia Lamblia causes microvilli atrophy, lymphocyte infiltration, and an increase in mucus secretion in the duodenum (Hasan et al.,1981, Marsh,1992, Öztürk et al., 2000). Although hematoxylin-eosin sections are often sufficient in duodenal pathologies, it may be necessary to support the diagnosis with histochemical studies when gastric metaplasia and or giardia considers. Staining with PAS, which does not disappear with d-PAS, shows gastric metaplasia; Giardia detects by staining pink-red with Masson Trichrome. And also, fragmented Brunner glands can be mistaken for myxoid nerve sheath tumors. It is easy to distinguish Brunner glands by showing reactivity with PAS (Garvey et al., 1992; McManus et al., 1960). Duodenal histochemical staining has uses in applications to cases where this is unsure but suspicious on hematoxylin-eosin sections. Still, there has been no study to assess the superiority of histochemical staining in duodenal pathology compared to hematoxylineosin sections. Our research aims to understand whether the routine use of histochemical stains in duodenal pathologies would provide power in diagnosis compared to hematoxylin-eosin sections. 


\section{MATERIALS AND METHODS}

Our study is a retrospective study conducted in 2011 using the block archives of 2001-2010. According to the 2011 ethical standards of the national clinical research committee based on the Declaration of Helsinki, no formal authorization requires for such retrospective studies. That is, already existing archival preparations use Because the materials of the patients who have their consent and information on the pathological examination senses to the laboratory - the data usage permission of Van Yüzüncü Yıl University Medical faculty patients reserves in the patient document archive.

By the ethical standards of the 2011 national clinical research committee, the slide archives of 110 patients retrospectively diagnosed with Gluten Enteropathy, 50 Duodenitis, and ten normal histological duodenal mucosae between 2001 and 2010 re-evaluate under the light microscope. In addition, histochemical staining was applied to pathological archive preparations to see the advantages of routine histochemical staining in diagnosis. According to Marsh criteria, those diagnosed with gluten enteropathy were examined, and those diagnosed with duodenitis were re-examined for the presence of epithelial erosion, blunting of the villi, lamina propria, and intraepithelial inflammatory infiltration.

We evaluated thickening in the subepithelial collagen layer and increased collagenization in the submucosa, as in collagenous colitis and giardiasis in differential diagnosis with Masson Trichrome stain. We reviewed the preparations in terms of gastric metaplasia with PAS and d-PAS. We compared the staining patterns in duodenitis and gluten enteropathy with PAS-AB and HID-AB in normal histological duodenal mucosa. We examined it with hematoxylin-eosin sections.

We used the Chi-square test and the likelihood-ratio test to determine the relationships between categorical variables. Besides, multiple coherence analysis was performed to determine the relationships between categorical variables together. Statistical significance level takes like 5\% (Ps 0.05) in calculations, and SPSS (ver:13) statistics package program was used for analyses.

\section{RESULTS AND DISCUSSION}

When 50 gluten enteropathy and 50 duodenitis cases were re-evaluated in light microscopy, they were compared in terms of flattening and blunting of the villi, crypt hyperplasia, intraepithelial lymphocyte count, lymphoplasmacytic infiltration in the lamina propria, intraepithelial neutrophilic infiltration, erosion of the surface epithelium, Brunner gland hyperplasia, goblet count per 100 epithelium and giardia. Ten duodenum cases with normal histological features were also evaluated with these parameters because we performed the histological evaluation of our patients in terms of Marsh criteria used to diagnose gluten enteropathy.

It was remarkable that the number of goblets in 100 epithelium and intraepithelial neutrophilic infiltration did not differ for all three groups. ( $P=0.176$ ve $\mathrm{P}=0.096$ ) Intraepithelial lymphocyte count was observed as minimal 41 in patients with gluten enteropathy. However, it was observed that this infiltration was 37 in cases with duodenitis. Intraepithelial lymphocyte infiltration does not observe in the duodenums with normal histological properties. As shown in Table 2, a significant variation was found in the intraepithelial lymphocyte count among the Gluten enteropathy, duodenitis, and normal duodenum $(P=0.010)$.

Erosion of the surface epithelium was focal in one (10\%) of the duodenal cases with normal histological features, was diffuse in 6 of the patients with gluten enteropathy, and focal in 20 (54\%) of the issues with gluten enteropathy. In duodenitis, 
diffuse erosion observes as one and focal erosion as 20 (42\%). While detection of corrosion in normal mucosal epithelium less than others and $61.7 \%(P=0,002)$ in patients with villi flattening, $62.7 \%(P=0.000)$ in patients with blunting of the villi, $57.9 \%$ $(P=0.046)$ in those with significant lymphoplasmacytic infiltration in the lamina propria indicated that the determined histopathological findings triggered erosion; none of them could identified as the primary etiological cause. What is noteworthy was the increased frequency in patients with gluten enteropathy $(P=0.034)$.

It was significant that flattening and blunting of the villi were observed in 74$80 \%$ of patients with gluten enteropathy, $20 \%$ of patients with duodenitis, and not seen in the duodenum with normal histological features $(P=0.000)$. Although crypt hyperplasia sees in only $33.6 \%$ of total patients, it was remarkable that all of them were in patients with Gluten enteropathy $(\mathrm{P}=0.000)$. Brunner gland hyperplasia also observes in $3.6 \%$ of the total number of patients, and it also sees in all patients with Gluten enteropathy $(P=0.000)$. Also, while a significant relationship was finds between crypt hyperplasia and villus flattening, villus blunting, and lymphoplasmacytic infiltration parameters in the lamina propria, it was seen that there was no connection between crypt hyperplasia and erosion and Brunner gland hyperplasia ( $P=0.067$ and $\mathrm{P}=0.074$, respectively). However, it was remarkable that Brunner gland hyperplasia observes with a rate of $100 \%$, with a markedly increased lymphoplasmacytic infiltration in the lamina propria $(\mathrm{P}=0.020)$.

As seen in Table 3, it was found that a slight increase in lymphoplasmacytic infiltration in the lamina propria was frequently (90.9\%) in normal histology, moderate growth was frequently $(52.5 \%)$ in patients with gluten enteropathy, and a significant increase was often (55.3\%) in patients with duodenitis $(P=0.000)$. Of the gluten enteropathy cases, 20 were considered as Marsh type 3c (40\%), 8 were Marsh type 3b (16\%), 4 were Marsh type 3a (8\%), 6 were Marsh type $2(12 \%), 12$ were Marsh type $1(\%)$. It was remarkable that the cases were mostly Marsh types $3 \mathrm{c}$ and 1 . While total atrophy observes in 20 of the cases diagnosed with gluten enteropathy according to Marsh criteria, atrophy find to be independent of intraepithelial lymphocyte count.

Histochemical studies first evaluated PAS and d-PAS and gastric metaplasia. Since gastric metaplasia does not occur detects in our sample groups, no relationship was found between gastric metaplasia and duodenitis, Gluten enteropathy, and normal histological duodenal mucosa. Masson Trichrome did not detect giardia, thickening of subepithelial collagen, and increase in mucosal collagen-fibrosis in all three groups.

Both gastric metaplasia and reactive epithelial changes in the three groups were targeted with PAS-AB and HID-AB. Gastric metaplasia was not observed in all three groups with $P A S-A B$, while purple-blue staining regards in goblet cells and purple in Brunner glands. Blue staining celebrates in goblet cells without any difference in the three groups, consistent with the normal small bowel epithelial staining pattern with HID-AB. 
Table 1. Modified Marsh Classification of Duodenal Biopsies

\begin{tabular}{llll}
\hline Type & $\begin{array}{l}\text { IEL per 100 } \\
\text { enterocytes }\end{array}$ & Crypts & Villus \\
\hline 0 & $<40$ & Normal & Normal \\
1 & $>40$ & Normal & Normal \\
2 & $>40$ & Increased & Normal \\
$3 \mathrm{a}$ & $>40$ & Increased & Mild atrophy \\
$3 \mathrm{~b}$ & $>40$ & Increased & Significant atrophy \\
$3 \mathrm{c}$ & $>40$ & Increased & None \\
\hline
\end{tabular}

Table 2. Relationship between Intraepithelial Parameters and Study Groups

\begin{tabular}{llcccccc}
\hline & Group & Median & Avg. & St. Dev. & Min. & Max. & $p$ \\
\hline Intraepithelial & Gluten & 47,00 & 50,92 & 10,58 & 41,00 & 80,00 & \\
$\begin{array}{l}\text { Lymphocyte } \\
\text { infiltration }\end{array}$ & Duodenit & 17,50 & 18,20 & 9,01 & 2,00 & 37,00 & \\
& Normal & 0,00 & 0,00 & 0,00 & 0,00 & 0,00 & \\
& & & & & & & \\
I00 epithelium & Gluten & 7,00 & 8,56 & 5,30 & 2,00 & 35,00 & \\
goblet & Duodenit & 9,00 & 10,00 & 3,81 & 1,00 & 19,00 & \\
number of cells & & & & & & & 0.176 \\
& Normal & 11,00 & 10,80 & 3,74 & 4,00 & 17,00 & \\
& & & & & & & \\
Intraepithelial & Gluten & 0,00 & 1,88 & 3,70 & 0,00 & 15,00 & \\
$\begin{array}{l}\text { Neutrophilic } \\
\text { infiltration }\end{array}$ & Duodenit & 1,00 & 2,50 & 3,25 & 0,00 & 14,00 & 0.096 \\
\hline
\end{tabular}

${ }^{*} \mathrm{p}$-value have significant effects

This test results from multiple coherence analysis 
Table 3. Intergroup Relationship with Lymphoplasmacytic Infiltration in the Lamina Propria

\begin{tabular}{|c|c|c|c|c|c|c|}
\hline & & & & Gro & & Total \\
\hline & & & Gluten & Duodenit & Normal & \\
\hline Lamina & Light & Count & 1 & 0 & 10 & 11 \\
\hline & & $\begin{array}{l}\text { \% within } \\
\text { Lamina }\end{array}$ & $9,1 \%$ & $0,0 \%$ & $90,9 \%$ & $100,0 \%$ \\
\hline & & $\begin{array}{l}\% \text { within } \\
\text { Group }\end{array}$ & $2,0 \%$ & $0,0 \%$ & $100,0 \%$ & $10,0 \%$ \\
\hline & & $\%$ of Total & $0,9 \%$ & $0,0 \%$ & $9,1 \%$ & $10,0 \%$ \\
\hline & Middle & Count & 32 & 29 & 0 & 61 \\
\hline & & $\begin{array}{l}\text { \% within } \\
\text { Lamina }\end{array}$ & $52,5 \%$ & $47,5 \%$ & $0,0 \%$ & $100,0 \%$ \\
\hline & & $\begin{array}{l}\text { \% within } \\
\text { Group }\end{array}$ & $64,0 \%$ & $58,0 \%$ & $0,0 \%$ & $55,5 \%$ \\
\hline & & $\%$ of Total & $29,1 \%$ & $26,4 \%$ & $0,0 \%$ & $55,5 \%$ \\
\hline & Prominent & Count & 17 & 21 & 0 & 38 \\
\hline & & $\begin{array}{l}\text { \% within } \\
\text { Lamina }\end{array}$ & $44,7 \%$ & $55,3 \%$ & $0,0 \%$ & $100,0 \%$ \\
\hline & & $\begin{array}{l}\text { \% within } \\
\text { Group }\end{array}$ & $34,0 \%$ & $42,0 \%$ & $0,0 \%$ & $34,5 \%$ \\
\hline & & $\%$ of Total & $15,5 \%$ & $19,1 \%$ & $0,0 \%$ & $34,5 \%$ \\
\hline Total & & Count & 50 & 50 & 10 & 110 \\
\hline & \% within Le & amina & $45,5 \%$ & $45,5 \%$ & $9,1 \%$ & $100,0 \%$ \\
\hline & $\%$ within G & roup & $100,0 \%$ & $100,0 \%$ & $100,0 \%$ & $100,0 \%$ \\
\hline & $\%$ of Total & & $45,5 \%$ & $45,5 \%$ & $9,1 \%$ & $100,0 \%$ \\
\hline Chi & are Tests & & & & & \\
\hline & & & Value & $d f$ & & $\begin{array}{l}\text { hallow. } \\
\text { led) }\end{array}$ \\
\hline Pearsol & Chi-Square & & $99,725(a)$ & 4 & & \\
\hline Likeliho & od Ratio & & 62.274 & 4 & & \\
\hline
\end{tabular}

Figure: 1 Histopathological image in duodenal biopsies
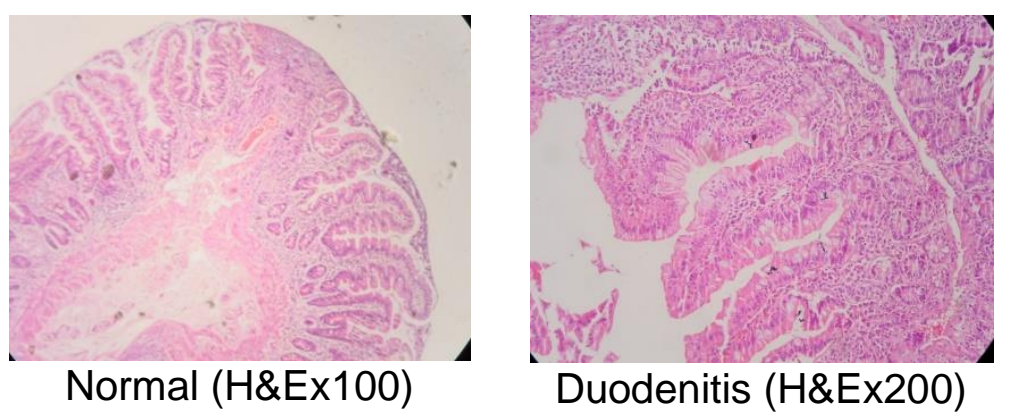

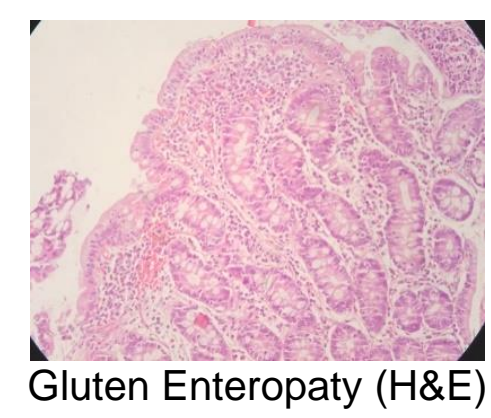


Figure: 2 Histochemical Stains in the Diagnosis of Gastric Metaplasia and Giardiasis

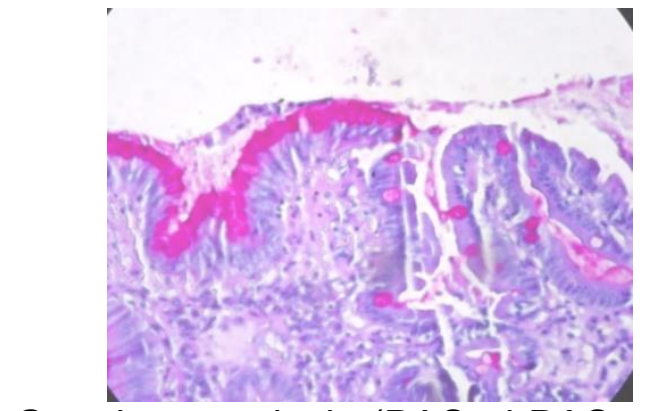

Gastric metaplasia (PAS, d-PAS x400)

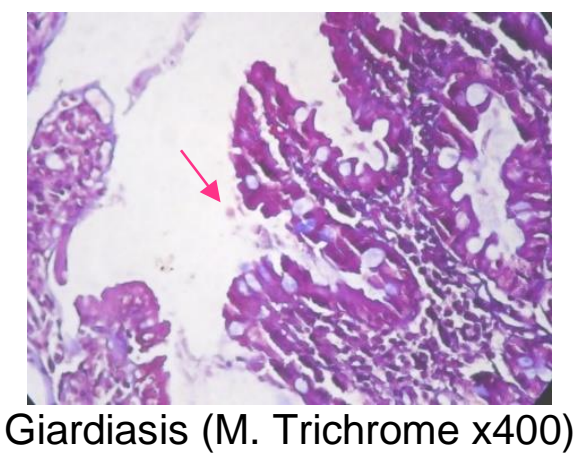

Figure: 3 Histochemical Staining Patterns Observed in Our Cases

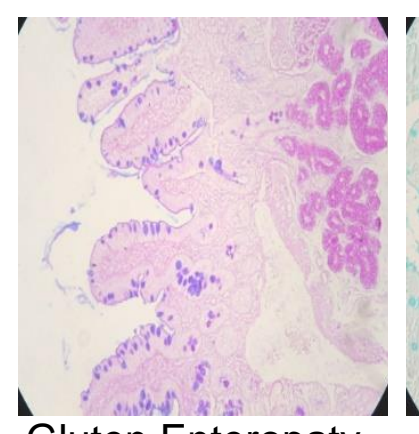

Gluten Enteropaty (PAS-ABx200)

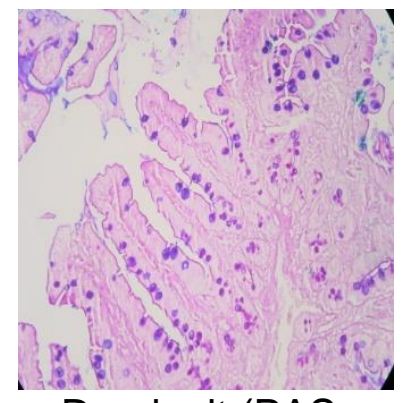

Duodenit (PAS$A B \times 200)$

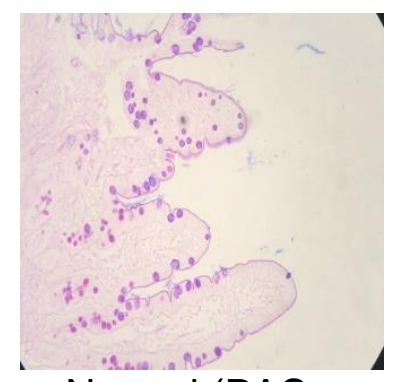

Normal (PAS$\mathrm{AB} \times 200)$

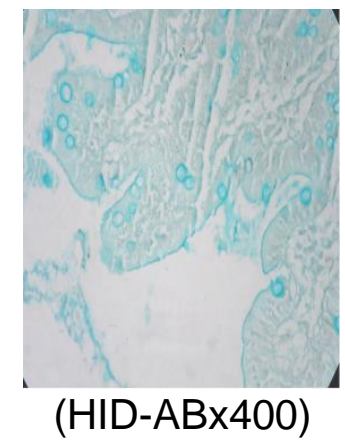

(HID-ABx400)

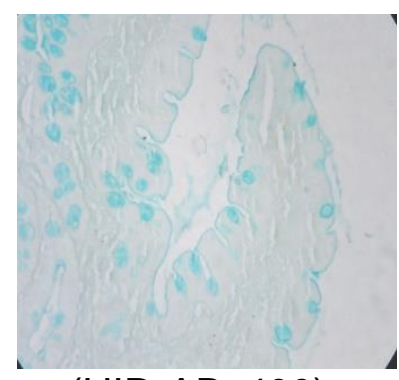

(HID-ABx400)

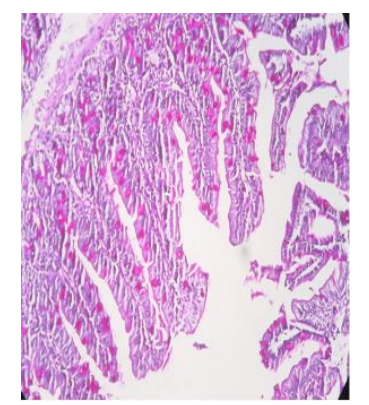

(PAS, $d-$ PAS 200$)$

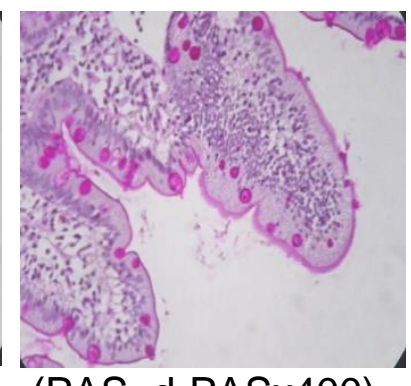

(PAS, d-PAS $\times 400)$

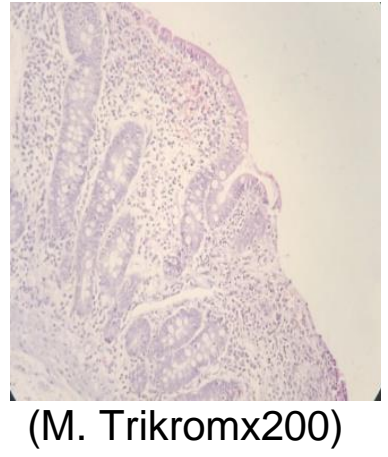

(M. Trikrom×200)

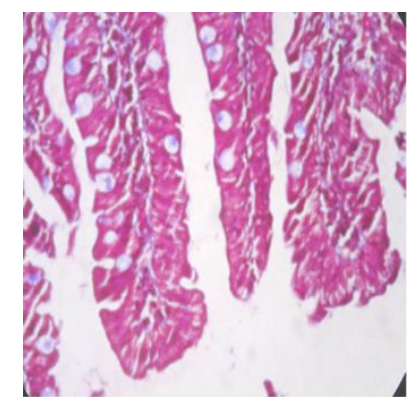

(M. Trikromx400) 
Since the loss of duodenal functions causes abdominal pain, bloating, diarrhea, indigestion, weight loss, anemia, osteoporosis, delayed puberty, depression, and infertility, it is vital to examine duodenal biopsies histopathologically. Clinical symptoms that may occur in duodenal pathologies are essential. We wanted to evaluate the contribution of histochemical stains to the diagnosis stage in duodenal biopsies, as they will affect the person's quality of life. Tissues are alive as soon as they leave the human body and exhibit the morphological findings of the disease they carry (if any). Formalin $(\mathrm{CH} 2(\mathrm{OH}) 2)$, which is a $10 \%$ solution of formaldehyde gas $(\mathrm{H} 2 \mathrm{CO})$ in water, is frequently used for fixation to prevent the current appearance of the tissues from changing and deteriorating with the effect of heat, humidity, and enzymes. Microscopic details are observed more clearly in the detected tissues (Figen, 2005).

Hematoxylin-Eosin is a dye combination that routines use for histopathological examinations. Hematoxylin stains the nucleus in cells in blue-purple, and Eosin stains the cytoplasm in pink, allowing us to obtain information from the microscopy of cells and tissues (Luna, 1968). Intraepithelial lymphocyte infiltration (IEL) is a critical feature in gluten enteropathy histopathology (figure 1). Histopathological evaluation of mucosal changes makes according to the current most valid classification system (Table 1), which Marsh defined in 1992 (Marsh,1992 and Öztürk et al., 2000).

Giardia lamblia has a faint appearance in hematoxylin-eosin. Staining the Giardia by Masson Trichrome in pink-red and mucin in blue is very useful in detecting giardiasis (Figure 2) (Ament, 1972). Histochemical techniques can demonstrate metaplastic and reactive changes, bacteriological and parasitological diseases. Periodic acid Schiff is useful for glycogen, mucin, mucoprotein, glycoprotein, collagen, basement membranes, capsules, and blood vessels. Predigestion with amylase removes glycogen staining (diastase-PAS). There is no loss of PAS reactivity with dPAS in gastric metaplasia foci evident with PAS (figure 2). Although it is possible to distinguish duodenal pathologies histopathologically, histochemical studies may be required to confirm the diagnosis in the presence of gastric metaplasia, mucosal collagenization, and giardia.

In their histochemical study with Giemsa, they aimed to determine the incidence of $H$. pylori in duodenal ulcer, duodenitis, non-ulcer dyspepsia, and control groups with gastric metaplasia. In the absence of gastric metaplasia, no organisms were detected. In the presence of gastric metaplasia, the presence of $H$ Pylori was observed only in duodenitis and duodenal ulcer (Forbes et al., 1994). Our study thought that we could detect a difference in the incidence of gastric metaplasia for the three groups using routine histochemical methods. We could not see the superiority of histochemical methods in gastric metaplasia between groups, as we could not detect gastric metaplasia within the three groups. In other words, we found that routine PAS and dPAS use did not contribute to the diagnosis of gastric metaplasia and the differential diagnosis between our groups.

In their study, Ilyas Tuncer and İran Bayram used tissue transglutaminase expression and antitransglutaminase immunohistochemical stain on duodenal biopsies of normal and gluten enteropathy patients. They saw that it stained the muscularis mucosa and pericryptic fibroblasts. Since this staining pattern was the same in both groups, they found that it was not suitable for diagnosing gluten enteropathy with antitransglutaminase staining (ilyas et al., 2003). We thought that if we used Masson's Trichrome routinely, we could obtain a significant difference between the three groups in terms of subepithelial collagen thickening and mucosal collagenization. However, we did not see collagenization in the three groups, and we 
obtained similar results. Like the study of Illyas et al., routine staining with Masson Trichrome did not differ in the differential diagnosis for the three groups.

Igor et al. found giardia stains with c-kit (CD117) immunohistochemical staining in 14 patients with giardia and c-kit in all patients (Igor et al., 2009). C-kit is an immunohistochemical study. While we aimed to detect Giardia with Masson Trichrome staining, histochemical staining, and detect a difference in Giardia incidence between our study groups by routine histochemical study, we did not notice any difference for the three groups.

Rajaguru et al. indicated that the number of dendritic cells expressing with autophagy protein LC3 was high in the pre-treatment period and less after-treatment biopsies. Also, after-treatment biopsies with Masson Trichrome staining demonstrated a significant decrease in basement membrane thickness along with the treatment (Rajaguru et al., 2013). Although this study, which found a reduction in basement membrane thickness with Masson's Trichrome after treatment, made us think we would find differences in basement membrane thickness between the three groups, we found similar staining patterns in the three groups. We did not consider the posttreatment period.

Amani Mubarak et al. showed that CD3 staining has an importance in the histopathological findings of gluten enteropathy. Their study reported that CD3 immunohistochemical staining could identify gluten enteropathy when there is an inconsistency between serology and hematoxylin-eosin section results (Amani et al., 2015). Iftikhar et al. calculated an almost similar amount of intraepithelial lymphocytes with hematoxylin-eosin sections and immunostain CD3 and CD20 in Celiac disease (Iftikhar et al., 2016).

Fernando Arévalo Suárez et al. tried to reveal immunochemical differences in their study between Celiac disease and negative serology villous atrophy patient groups. They found that CD8, CD4, and CD56 lymphocytes were not different in these groups, and also numerous CD3 lymphocytes were shown in negative serology villous atrophy patients (Suáres et al., 2016). Prasenjit et al. could not find significant differences in anti-tissue Transglutaminase 2 immunostaining between Celiac disease, nonspecific duodenitis, and inflammatory bowel disease (Prasenjit et al., 2018)

CD3, CD20, CD8, CD4, CD56 and anti-tissue Transglutaminase 2 are immunostains. We did not use immunostaining in our study, as we wanted to evaluate the privileges that histochemical studies in the duodenum would give us in routine practice. Different results could not be obtained in our study between histochemical staining and normal duodenum, duodenitis, and gluten enteropathy in terms of differential diagnosis. Masson Trichrome did not detect giardia, thickening of subepithelial collagen, and increase in mucosal collagen-fibrosis in all three groups. While gastric metaplasia was not observed with PAS-AB in all three groups, it was stained purple in duodenal columnar epithelium and goblet cells. Blue staining in both the columnar epithelium and goblet cells did not differ in the three groups, consistent with the normal small bowel epithelial staining pattern with HID-AB. Brunner glands stains with PAS and d-PAS (Figure 3).

\section{CONCLUSION}

The present findings conclude that hematoxylin-eosin sections are more valuable in differential diagnosis than histochemical staining in duodenal biopsies. Studies on histochemical applications in terms of differential diagnosis or contribution 
to diagnosis in duodenal pathologies are few. Our research data is essential in shedding light on new studies on this subject.

\section{ACKNOWLEDGEMENT}

I want to thank Professor Doctor İrfan Bayram, Doctor Mehtap Eroğlu, and Doctor Elif Acar for their support in editing the article. This article produced from the thesis named 'Duodenal biopsies which particularly in the diagnosis of gluten enteropathy and various duodenal pathologies and compared about histochemical execution' which made according to the criteria of Helsinki Declaration.

\section{CONFLICT OF INTEREST}

The authors declare that they have no conflicts of interest.

\section{REFERENCES}

Amani, M., Victrorien, M.W., Roderick, H.J.H., Fiebo, J.W. ten K. (2015, Jun 28). Immunohistochemical CD3 staining detects additional patients with celiac disease. World J Gastroenterol, 21(24): 7553-7.

Ament, M.E. (1972). Diagnosis and Treatment of giardiasis, J. Pediatr.;80:633-7.

Eichinger, D. (2001). Encystation in parasitic protozoa. Curr. Opin. Microbiol, 4: 4216.

Farrell, R.J., Kelly, C.P. (2002). Celiac sprue and refrectory sprue. In Sleisenger \& Fordtran's Gastrointestinal and Liver Disease Pathophysiology/ Diagnosis/ Management, 7th ed. M Feldman, LS Friedman, MH Sleisenger (eds). Philadelphia. Elsevies Science; Vol II: 1818-41.

Figen, D. (2005). Histokimya. Aegean Pathology Journal, 2: 62-70.

Forbes, G.M, et al.(1994). Duodenal ulcer treated with Helicobacter Pylori eradication: seven year follow up. Lancet, 343(8892): 258-60.

Garvey, W., et al. (1992). Combined modified periodic acid-Schiff and batch staining method. J. Histotechnol, 15: 117-20.

Gillin, F.D., Reiner, D.S., McCaffery, J.M. (1996). Cell biology of the eukaryote Giardia Lamblia. Annu. Rev. Microbiol, 50: 679-705.

Hasan, M., Sircus, W., Ferguson, A. (1981). Duodenal mucosal architecture in nonspecific and ulcer-associated duodenitis. Gut, 22: 637-41.

Iftikhar, R., Jamal, S., Zafar, A., Saadia, A. (2016, Oct). Histopathological and Immunohistochemical Analysis of Small Intestinal Biopsies in Adults Suspected of Celiac Disease. J Coll Physicians Surg Pak, 26(10): 827-30.

Igor, S., Netta, S., Ruthy, S.L. (2009). C kit/CD 117 immünstain is useful for the diagnosis of giardia lamblia in duodenal biopsies. Human Pathology, 40(3): 3235.

Illyas, T., İrfan, B., İsmet, K., Ridvan, M., Serdar, U. (2003). Normal ve gluten enteropatili hastaların duodenal mukozasında doku transglutaminaz ekspresyonu. The Turkish J Gastroenterol, 14(3):185-8.

Johnson, L.R. (2013). Gastrointestinal Physiology, 8th edition. Phileladelphia. Elsevier/Mosby. ISBN978-0-323-10085-4.

Luna, L.G. (1968). Manual of Histologic Staining Methods of the Armed Forces Institute of Pathology. 3rd Edition, McGraw-Hill, New York.

Marsh, M.N. (1992). Gluten, major histocompatibility complex, and the small intestine. A molecular and immunobiologic approach to the spectrum of gluten sensitivity. Gastroenterology, 102: 330-54. 
McManus, J.F.A. and Mowry, R.W. (1960). Staining Methods Histologic and Histochemical, Paul B. Hoeber, New York;126-8.

Nilsen, E.M., Jahnsen, F.L., Lundin, K.E., et al. (1998). Gluten induces an intestinal cytokine response strongly dominated by interferon gamma in patients with celiak disease. Gastroenterology, 115: 551-63.

Noble, J. and Harry L.G. (2001). Greene, eds. Textbook of Primary Care Medicine, 3rd ed. St. Louis: Mosby-Year Book, Inc.

Öztürk, S., Serinöz, E., Keklik, T. et al. (2000). Histopathological assessment in cases with gluten-sensitive enteropathy. Significance of typing. The Turkish $J$ Gastroenterol, 11: 150-4.

Parasites and Health: Giardiasis. (2006, 20 May ). CDC website.

Prasenjit, D., Ramakant, R., Anil, K.V., Geetika, S., Archana, G.V., Rajni, Y., et al. (2018, Jul) Immunohistochemical Expression of Antitissue Transglutaminase 2 in Tissue Injuries: An Interpretation Beyond Celiac Disease. Appl Immunohistochem Mol Morphol, 26(6): 425-30.

Przemioslo, R.T., Lundin, K.E., Sollid, L.M., et al. (1995). Histological changes in small bowel mucosa induced by gliadin sensitive T lyphocytes can be blocked by antiinterferon gamma antibody. Gut, 36: 874-9.

Rajaguru, P., Vaiphei, K., Saikia, B., Kochhar, R. (2013). Increased accumulation of dendritic cells in celiac disease associates with increased expression of autophagy protein LC3. Indian J pathol microbiol, 56(4): 342-8.

Sanders, D.S., Hurlstone, D.P., Stokes, R.O., Rashid, F., Milford-Ward, A., Hadjivassiliou, M., et al. (2002, Jan) Changing face of adult coeliac disease: experience of a single university hospital in South Yorkshire. Postgrad Med J, 78(915): 31-3.

Suáres, F.A., Portugal, S., Barreda, C., Montes, P., Perez-Narrea, M.T., Rodriguez, O., et al. (2016, Apr-Jun) Celiac disease and negative serology villous atrophy: histological comparison and imunohistochemical study of CD3, CD4, CD8 and CD56 lymphocytes. Rev Gastroenterol Peru, 36(2): 123-8. 